\title{
EVOLUTION AND STATE OF THE LOCAL ISM
}

\author{
T. SCHMUTZLER ${ }^{1}$ AND D. BREITSCHWERDT ${ }^{2}$ \\ ${ }^{1}$ Institut für Theoretische Astrophysik, \\ Im Neuenheimer Feld 561, D-69120 Heidelberg, Germany \\ ${ }^{2}$ Max-Planck-Institut für Kernphysik, \\ Postfach 1039 80, D-69029 Heidelberg, Germany
}

The most puzzling observations concerning the LISM (distance $<100 \mathrm{pc}$ ) can be explained by a fast adiabatically cooled gas in the cavity of an old superbubble. The ultrasoft X-ray background and contributions to the Cand $\mathrm{M}$-bands are due to the continuum emission of delayed recombination [1]. In contrast to collisional ionization equilibrium (CIE) models, but consistent with recent observations [2], our model predicts a lack of emission lines and a low emissivity in the EUV range. In the figure below we compare the emissivities resulting from CIE at $T=10^{6} \mathrm{~K}$ and those from our model at $T=4.2 \times 10^{4} \mathrm{~K}$. The basic feature of our model is a thermally self-consistent approach of the time-dependent evolution.

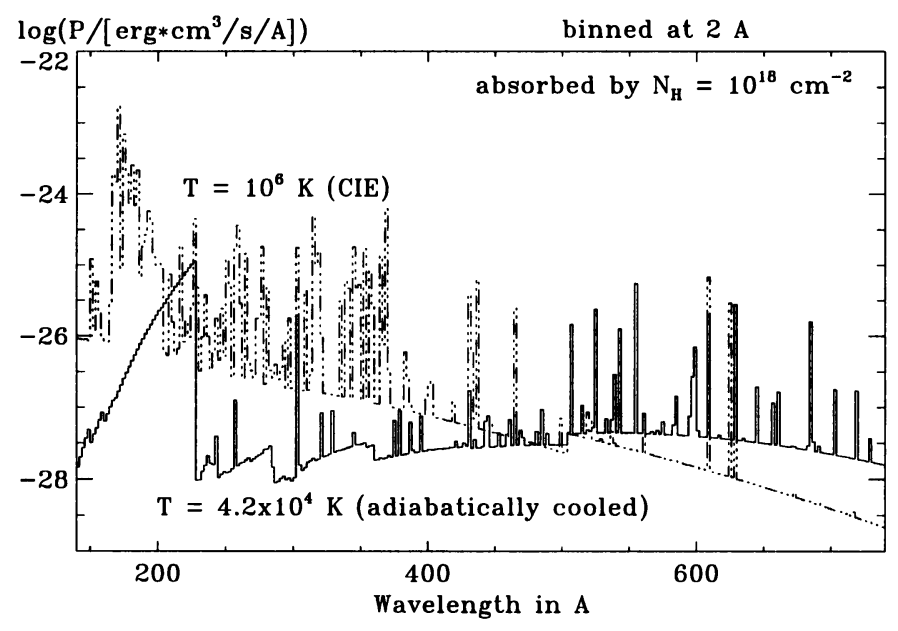

\section{References}

1. Breitschwerdt, D., Schmutzler, T., 1994, Nature, 371, 774-777

2. Jelinsky, P., Vallerga, J.V., Edelstein, J., 1995, Astrophys. J., 442, 653-661 